\title{
Anisotropic Interaction and Motion States of Locusts in a Hopper Band
}

\author{
Jasper Weinburd ${ }^{1, \dagger}$, Jacob Landsberg $^{2}$, \\ Anna Kravtsova ${ }^{1}$, Shanni Lam ${ }^{1}$, Tarush Sharma ${ }^{1}$, \\ Stephen J Simpson ${ }^{3,4}$, Gregory A Sword ${ }^{5}$, and Jerome Buhl ${ }^{6}$ \\ ${ }^{1}$ Department of Mathematics, Harvey Mudd College, Claremont, CA, 91711, USA \\ ${ }^{2}$ Department of Physics and Astronomy, Haverford College, Haverford, PA, 19041, USA \\ ${ }^{3}$ School of Life and Environmental Sciences, University of Sydney, Sydney, New South Wales 2006, Australia \\ ${ }^{4}$ Charles Perkins Centre, University of Sydney, Sydney, New South Wales 2006, Australia \\ ${ }^{5}$ Department of Entomology, Texas A\&SM University, College Station, TX, 77843, USA \\ ${ }^{6}$ School of Agriculture, Food and Wine, University of Adelaide, Adelaide, Southern Australia 5005, Australia
}

${ }^{\dagger}$ Corresponding author: jweinburd@hmc.edu

December 16, 2021

\begin{abstract}
Swarming locusts present a quintessential example of animal collective motion. Juvenile locusts march and hop across the ground in coordinated groups called hopper bands. Composed of up to millions of insects, hopper bands exhibit coordinated motion and various collective structures. These groups are well-documented in the field, but the individual insects themselves are typically studied in much smaller groups in laboratory experiments. We present the first trajectory data that detail the movement of individual locusts within a hopper band in a natural setting. Using automated video tracking, we derive our data from footage of four distinct hopper bands of the Australian plague locust, Chortoicetes terminifera. We reconstruct nearly twentythousand individual trajectories composed of over 3.3 million locust positions. We classify these data into three motion states: stationary, walking, and hopping. Distributions of relative neighbor positions reveal anisotropies that depend on motion state. Stationary locusts have high-density areas distributed around them apparently at random. Walking locusts have a low-density area in front of them. Hopping locusts have low-density areas in front and behind them. Our results suggest novel interactions, namely that locusts change their motion to avoid colliding with neighbors in front of them.
\end{abstract}

\section{Introduction}

Locust hopper bands exhibit a striking example of collective motion in insects. Without a complex social structure, juvenile locusts self-organize into a range of patterns from columnar streams to planar fronts that appear to aid in ecological functions of the group, such as migration and foraging $[59,53]$. Similar collective motion is observed in schools of fish [41], flocks of birds [6, 40], and even herds of ungulates [58]. These natural phenomena have produced a field of research centered on the idea that a group can attain collective goals without centralized instruction.

Early minimal models of collective motion were introduced and developed by computer scientists [44], physicists [60], and mathematicians [17] and have remained largely unchanged. While varying in implementation or ideology, the modern descendants of these models universally rely on a few rules for simple interactions between individuals. These interactions are often characterized as attraction, repulsion, and alignment of the direction of motion. Empirical studies by biologists, ecologists, and physicists have provided evidence supporting these models and informing these interaction rules for specific species. For instance, Ballerini et al. [5] found a topological interaction network in 
flocking starlings. Katz et al. [38] documented nearest neighbor following behaviors in schooling fish. Lukeman et al. [40] calculated a characteristic repulsion distance for surface-swimming ducks. For locusts, Buhl et al. [14] revealed a maximal distance for pairs of conspecifics to align their motion.

Locusts and their motion are the subject of a great many studies ranging from empirical work in the lab $[12,7,9,2]$ or field $[16,59,23,33,14]$ to theoretical modeling $[21,19,3,11,27]$; for a review see the work of Ariel and Ayali [1]. Locusts exhibit phase polyphenism, a phenomenon whereby an individual can exhibit two distinct phases of behavior (and for some species also distinct morphologies). In the solitarious phase, locusts typically avoid each other and forage individually. Crowding by conspecifics triggers a transition to the gregarious phase in which individuals gather in social aggregations [43]. When composed of juveniles, these aggregations are called hopper bands because locust nymphs, whose wings have not fully developed, hop and walk across the ground. Hopper bands are often composed of hundreds of thousands or millions of locusts all moving as a collective $[16,59]$. The directions of motion of individuals are highly aligned and the band typically takes on one of a few distinctive shapes. In some species, such as the brown locust, Locustana pardalina, the shape of the group is highly stretched along the direction of motion, often appearing as dense and thin streams [59, 55]. For the Australian plague locust, Chortoicetes terminifera, a dense front is often observed [16, 33, 14], especially when the hopper band moves through thick grasses. These examples do not provide general rules; these shapes have been observed across species and in various environments. Many questions remain as to when a hopper band takes on a particular shape, what function these shapes play, and how they develop from the interactions among individuals. These questions lead researchers to explore the motion and shape of hopper bands through the lens of collective motion.

Relatively simple collective motion models have successfully reproduced collective patterns of hopper bands observed in the field. Dkhili et al. [19] demonstrated that both columns and fronts can be achieved by varying individual-level parameters in a model that incorporated only local interactions between locusts. A similar approach was taken by Bach [3], with a realistic number of individuals and parallel computing approach. An alternative approach by Bernoff et al. [11] incorporated individual locust interactions with food resources and showed that dense fronts are typical when sufficient food is present. Modelers often rely on hypothesized interactions at the individual level. The most common assumption is the simplest; that individual interactions are isotropic, that is, interactions depend only on the distance between individuals and not on their relative positions. One notable exception is the escape-and-pursuit model [48], which hypothesizes that the primary driver of locust marching is tied to cannibalistic behavior [7, 8, 31] (chasing those in front and fleeing from those behind). In this modeling framework, locusts are likely to have neighbors directly in front or behind them [13] but this has not been observed empirically.

A majority of the empirical work studying the motion of hoppers is conducted through either laboratory experiments focused on individuals $[12,7,9,2,20]$ or field observations of the group as a whole [16, 59, 23, 33]. These field observations date back to Clark [16], Ellis and Ashall [22], and Uvarov [59] and are mainly qualitative in nature, for instance noting the shape of the hopper band at different times of day or in varying vegetation cover. Many laboratory studies are conducted by placing a relatively small number (less than a hundred) of locusts in an arena and observing their motion. With this technique, critical densities for the onset of ordered marching have been established [12]. By analyzing individual locust trajectories in an arena, a pattern of "stop-and-go" motion has been characterized and associated with collective motion [7, 9, 2]. Other arena studies examine the effect of cannibalism [12, 7, 8, 31] and the presence of food [20]. A recent arena study [39] found that marching with just nine other locusts triggered motion qualitatively different from isolated marchers. Individual-level quantitative information from within a hopper band in a natural setting is restricted to just a single set of data analyzed by Buhl et al. [14, 13]. Buhl et al. [13] found support for isometric interactions by computing neighbor densities that were rotationally symmetric around a focal individual. While all of these empirical studies have advanced our understanding of the mechanics of locust motion and interaction, there is still a particular dearth of data on individual interactions during collective motion within a group of a naturally occurring size.

We present and analyze the first trajectory data of individual locusts moving within a hopper band. We study four hopper bands of the Australian plague locust, Chortoicetes terminifera, from 
an outbreak in 2010 near Hillston, New South Wales, AUS. We recorded video of locusts moving across the ground using cameras mounted on tripods (see Sample video clips for a sample). Using automatic tracking software (TrackMate [57]) we extracted 19687 individual trajectories by linking 3369723 locust positions. Our analysis of these trajectories suggests that locusts adjust their motion to avoid neighbors ahead of them, providing evidence of a novel locust-locust interaction for collision avoidance in hopper bands. These results add to the understanding of social interactions in marching locusts and provide valuable insight for modelers seeking to reveal the mechanisms behind the collective motion of the swarm.

\section{Results}

Our data set was extracted from recordings of four bands of Australian plague locust, Chortoicetes terminifera. The data consists of 3369723 locust positions linked into 19687 trajectories from 24300 frames (twenty-seven minutes) of video. From these trajectories we inferred 3332137 heading directions and individual speeds; Figure 1 (left) shows a histogram of these speeds. We constructed a plot of the relative density around a focal individual from 19407719 nearby neighbor positions, shown in Figure 1 (right). The anisotropy apparent in this plot contrasts with previous findings by Buhl et al. [13] and motivates deeper investigation. To this end, we classified the data into distinct motion states (stationary, walking, and hopping) using statistical learning. We partitioned the relative neighbor data by motion state of the focal individual to reveal differences in anisotropy depending on motion.

Our results are divided into three sections. In the first we report indicators of collective marching which confirm that the locusts recorded in our videos are displaying collective motion. The second describes basic statistics of locust speed and motion state, providing estimates for the proportion of insects that are stationary, walking, and hopping. In the third, we quantify the anisotropy in the relative neighbor density distribution around a focal locust in each motion state. In the remainder of the paper, we often use "locust" to refer to the Australian plague locust (APL) specifically and acknowledge that some results may be species specific.
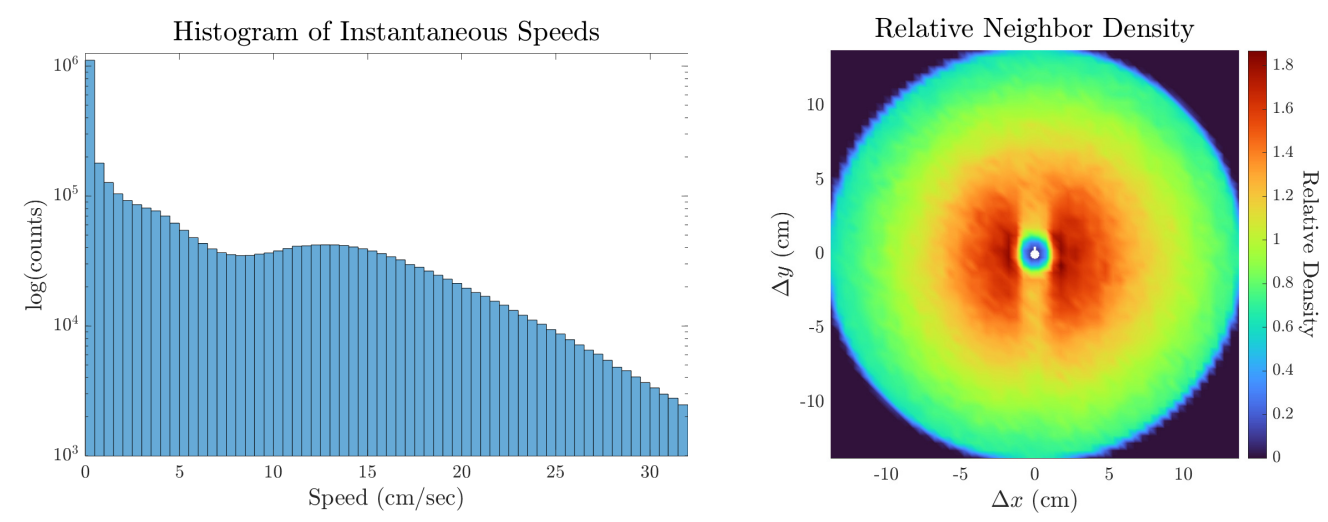

Figure 1: Histogram of speeds (left) and plot of relative neighbor density (right). The distribution of speeds (left) is bimodal, with peaks near 0 and $13 \mathrm{~cm} \mathrm{~s}^{-1}$. From approximately $2-5 \mathrm{~cm} \mathrm{~s}^{-1}$, the counts decrease exponentially (linear decrease on the logarithmic scale). The two peaks and exponential decrease reflect the hidden distributions of locusts in distinct motion states. The relative neighbor density (right) is computed from 19407719 relative neighbor positions around a representative focal locust (white marker) positioned in the center and oriented facing upwards, along the vertical axis. Density is highest (red) within a radius of $5 \mathrm{~cm}$ and lowest (blue) in a central disc with radius approximately $1 \mathrm{~cm}$. The density is not rotationally symmetric; at distances of $1-7 \mathrm{~cm}$ there is a noticeable decrease in density (orange) directly in front (above) and behind (below) the focal locust. At distances greater than $7 \mathrm{~cm}$, the density appears to be rotationally symmetric. Neighbors at distances greater than $14 \mathrm{~cm}$ were omitted. 

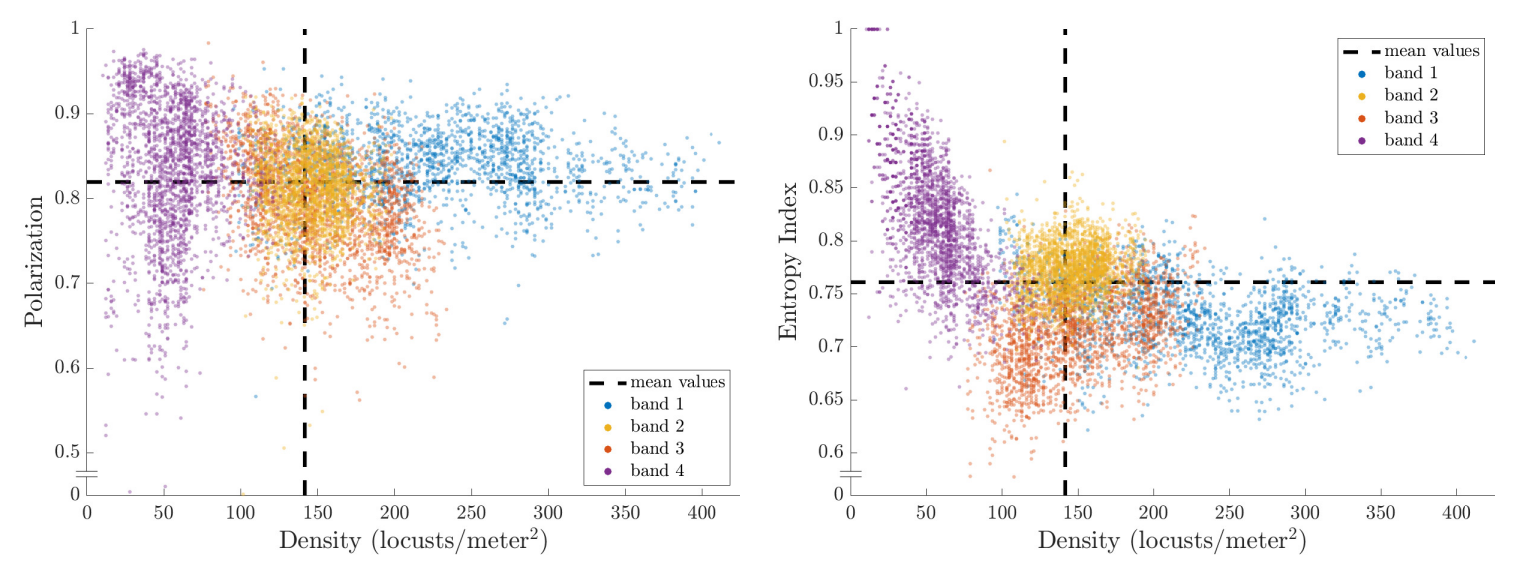

Figure 2: Measures of collective alignment plotted against density for all bands (distinguished by color). Polarization (left) is highly variable for densities less than 200 locusts $/ \mathrm{m}^{2}$. Entropy (right) shows a marked decrease with increasing density, consistent with [14]. Data plotted is a regular subsample of frames (every fifth frame), with mean values of the full data set (dashed lines). Essentially all measurements lie within ranges associated with collective marching for hopper bands.

\subsection{Collective marching}

All four bands exhibited collective marching behavior. The mean density across all recordings 141.6 locusts $/ \mathrm{m}^{2}$ is well above the established 20 locusts $/ \mathrm{m}^{2}$ threshold for marching [12]. Measurements of group alignment (polarization and entropy index) agree with previous measurements of marching locusts in the field [14]. In particular, we compute a mean polarization of 0.82 and mean entropy index of 0.76 across all bands.

We present values for each band in Appendix B (Table 2) and direct measurements for a regular subsample of frames (every fifth frame) as scatter plots in Figure 2. The density of each band is relatively well-cluster, as expected. There is a distinct decrease in entropy with increasing density and a high variance in polarization for densities below 200 locusts $/ \mathrm{m}^{2}$. See [14] for an in-depth analysis of similar trends. The variance in polarization is partly a symptom of its ambiguity as an order parameter and partly the result of our coarse measure of density as global in the whole field of view. For instance, our density does not well describe a small but highly-aligned group that is isolated in an otherwise empty frame of video. For higher densities, there is no space for such a group to be isolated so the local density will typically be closer to the global measure we have recorded.

We computed a mean speed of $6.5 \mathrm{~cm} \mathrm{~s}^{-1}$ and plot the distribution of all speeds in Figure 1 (left). Mean speeds for each band are recorded in Appendix B (Table 3). These values are not directly comparable to an established measurement. Measurements of speed for the APL in the field are typically collective band speeds, averaged over a matter of hours so that they may include various locust behaviors besides marching such as feeding or basking. Laboratory studies have quantified individual speed more directly with mean speeds of 1.5 and $3.2 \mathrm{~cm} \mathrm{~s}^{-1}$, which we inferred from the walking speed and proportion of moving locusts reported by Buhl et al. [12] and Bazazi et al. [7] respectively for Schistocerca gregaria. Our measurement falls in the same order of magnitude. The difference is likely due either to the smaller speeds being observed in a laboratory setting or that the desert locust, $S$. gregaria, appears to hop less often than the APL, C. terminifera, in our experience.

\subsection{Individual speed and motion state}

Of the 3369723 locust positions, we computed 3332137 individual speeds, presented in Figure 1 (left). (The discrepancy in the number of positions and speeds is due to the start and end of trajectories.) Each speed was classified into one of three motion states by a support vector machine. These motion states divide the data into $26.0 \%$ stationary, $24.7 \%$ walking, and $49.2 \%$ hopping. We computed mean speeds for each motion state and found $(2.0 \pm 0.7) \mathrm{cm} \mathrm{s}^{-1}$ (stationary), $(2.7 \pm 2.2) \mathrm{cm} \mathrm{s}^{-1}$ 

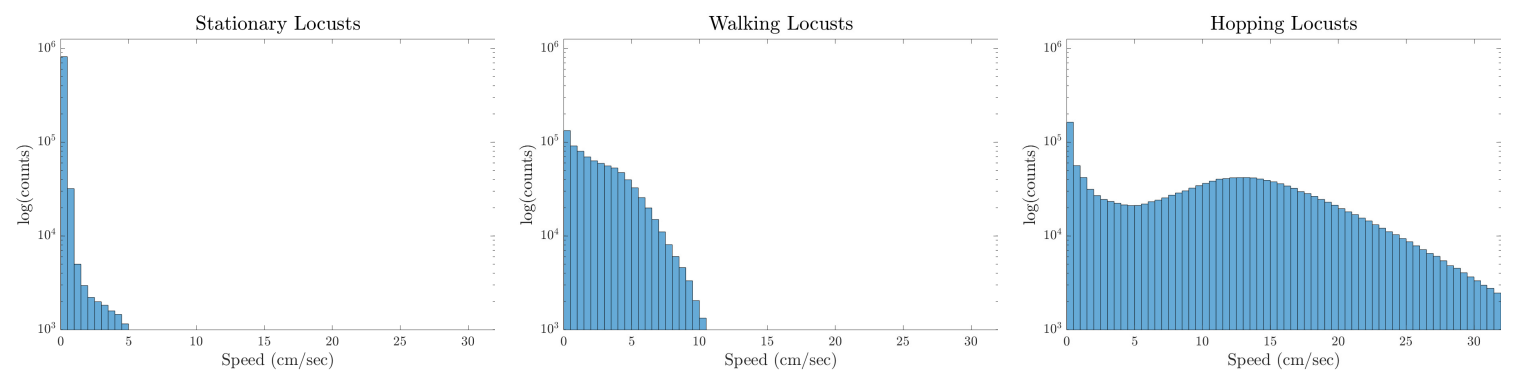

Figure 3: Histograms of speeds for stationary (left), walking (center), and hopping (right) locusts. All appear with the same log-scaled vertical axis. Matching intuition, the speeds of stationary locusts are tightly grouped near $0 \mathrm{~cm} \mathrm{~s}^{-1}$ - speeds above $1 \mathrm{~cm} \mathrm{~s}^{-1}$ make up less than $2.5 \%$ of all stationary data. Walking locusts have speeds between 0 and $10 \mathrm{~cm} \mathrm{~s}^{-1}$ with a steeper decline in numbers after $5 \mathrm{~cm} \mathrm{~s}^{-1}$. Hopping locusts have a bimodal distribution with one peak near 0 and the other near $13 \mathrm{~cm} \mathrm{~s}^{-1}$, as we expect from the pattern of pausing between hops that we observed in our video recordings.

(walking), and $(11.8 \pm 9.3) \mathrm{cm} \mathrm{s}^{-1}$ (hopping). Each mean speed has plus/minus one standard deviation. Motion state and speed data are presented for each band in Appendix B (Table 3). See Sample video clips for a visualization showing results of the motion state classification.

Figure 3 shows histogram plots for the speed distributions divided by motion state. Note the logarithmic scale on the vertical axis. Speeds of stationary locusts essentially all fall into the first two bins $0-1 \mathrm{~cm} / \mathrm{sec}$. Higher speeds make up less than $2.5 \%$ of all data classified as stationary. The number of walking speeds have no such dramatic peak near 0 , instead decreasing slowly until $5 \mathrm{~cm} \mathrm{~s}^{-1}$, then decreasing super-exponentially (concave down on the logarithmic plot). Speeds of hopping locusts are by far the most widely distributed with two peaks at 0 and $13 \mathrm{~cm} \mathrm{~s}^{-1}$, matching our observation that hopping locusts often pause between two jumps. A minimum around $5 \mathrm{~cm} \mathrm{~s}^{-1}$ separates these two maxima and the hopping speeds decrease super-exponentially after the second. Comparing these three plots to Figure 1 (left), we observe that our motion state assignment has cleanly divided the data into three distributions with unique features that were all visible in the full distribution.

\subsection{Anisotropy in relative neighbor density}

Figure 1 (right) demonstrates that the relative neighbor density is not isotropic, i.e. not rotationally symmetric, particularly at distances of 1-7 $\mathrm{cm}$ from the focal individual. At distances greater than $7 \mathrm{~cm}$, the density appears isotropic. We therefore focused next on a smaller square around the focal locust so as to exclude the isotropic region at larger radii. Additionally, we partitioned the relative positions of neighbors according to the motion state of the focal individual.

In Figure 4, we plot the relative neighbor densities around focal locusts that are stationary, walking, and hopping. In each plot, the focal locust is positioned in the center $(\Delta x=\Delta y=0)$ and faces upwards (along the $\Delta y$-axis). The highest relative neighbor densities are indicated by red, intermediate densities are shown in green, and the lowest neighbor densities appear in blue. For all three motion states, there is a roughly circular area of low density around the focal individual with a radius of approximately $1 \mathrm{~cm}$. Past a radius of $7 \mathrm{~cm}$, the plots are roughly isotropic (rotationally symmetric) and uniform between motion states.

The visual differences in relative density plots between motion states in Figure 4 are striking. For stationary focal locusts, the relative neighbor density is mostly isotropic, with localized spots of high density (red) distributed apparently at random. For walking locusts, there is a distinct area of lower density ahead of the focal individual. This void has the approximate shape of a $45^{\circ}$-sector. The highest relative neighbor densities are directly to the left and right of the focal individual at a distance just less than $2 \mathrm{~cm}$. For hopping locusts, there is a strip-shaped area of low density ahead and behind the focal individual. This void appears to divide an otherwise circular area of high 

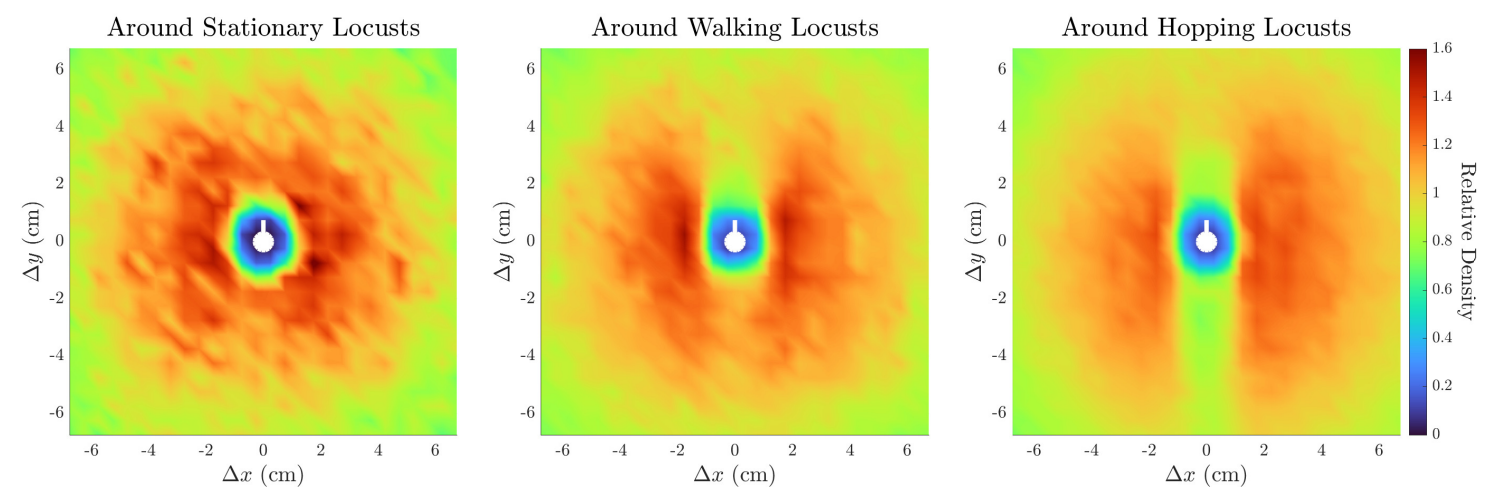

Figure 4: Relative neighbor density around locusts that are stationary (left), walking (center), and hopping (right). A representative focal locust (white marker) is positioned in the center of each plot and oriented facing upwards along the vertical axis. Areas of highest density (red) are distributed apparently at random around a stationary focal locust but highly anisotropic around walking and hopping focal locusts. A notable sector-shaped area of lower density (green-orange) lies immediately in front (above) the walking focal locust. A longer and narrower strip-shaped area of low density (green) lies in front and behind the hopping focal locust.

density that decreases with distance from the center. The high-density area to the right of the focal individual is larger than on the left, which we attribute to a density gradient in bands 1 and 3 . The observed low-density sector in front of walking locusts and the strip both in front and behind hopping locusts are novel anisotropies for neighbor densities in the APL and other locust species.

We quantified these anisotropies by examining the angular distribution of neighbors within $7 \mathrm{~cm}$. First, the Hodges-Ajne test for uniformity confirms that, for a focal locust in any motion state, the distribution of neighbor angles is not drawn from the uniform distribution of angles with $p$-values less than $10^{-10}$ (stationary), less than $10^{-128}$ (walking), and less than machine error (hopping). We characterize the degree of this nonuniformity by computing trigonometric moments, which we record in Appendix B (Table 4). Overall anisotropy $\left|\mathbf{M}_{p}\right|$ is significantly larger for the distributions around walking and hopping locusts $\left(\left|\mathbf{M}_{1}\right|=0.0173,0.0203\right.$ and $\left.\left|\mathbf{M}_{2}\right|=0.0248,0.0358\right)$ than around stationary locusts $\left(\left|\mathbf{M}_{1}\right|=0.0040\right.$ and $\left.\left|\mathbf{M}_{2}\right|=0.0146\right)$. This quantitatively confirms what Figure 4 shows visually - that the distributions around moving locusts are less uniform than around stationary locusts.

In Figure 4 we noted two forms of anisotropy around moving locusts. First, the sector of lower density in front of walking locusts is unimodal and therefore measured by $\mathbf{M}_{1}$. We compute the frontback asymmetry as $-M_{1}^{s}=\mathbf{M}_{1} \cdot\langle 0,-1\rangle$ to measure its size along the apparent axis of asymmetry (vertical). The negative captures the lower density in front and higher density behind. We point out the extreme disparity in $-M_{1}^{s}$ between the distributions around walking (0.0173) and stationary (0.0023) focal locusts. Second, both walking and hopping locusts have areas of high density on either side. This is measured by $\mathbf{M}_{2}$ and we compute the four-fold anisotropy $M_{2}^{c}=\mathbf{M}_{2} \cdot\langle 1,0\rangle$ to measure its size along the apparent axis of high density (horizontal). We highlight the extreme disparity in $M_{2}^{c}$ between the distributions around hopping (0.0357) and stationary $(0.0142)$ focal locusts. These values are reported in bold in Appendix B (Table 4), along with other detailed anisotropy quantities.

Finally, in Figure 5 we examined how front-back asymmetry $-M_{1}^{s}$ (dashed blue) and four-fold anisotropy $M_{2}^{c}$ (solid orange) depend on distance from the focal individual. We computed $-M_{1}^{s}$ and $M_{2}^{c}$ for subsets of neighbors from overlapping annuli of width $\Delta r=1 \mathrm{~cm}$ at intervals of $r=0.25 \mathrm{~cm}$. In each annulus, we scaled the anisotropy by the ratio of the density in that annulus to the density in the complete disc with radius $14 \mathrm{~cm}$. For a measure of distance from uniformity, we plotted the same quantities computed analytically for a uniform distribution. The uniform distribution of angles on the circle has $-M_{1}^{s}=M_{2}^{c}=0$ (black) and standard deviation $\frac{1}{\sqrt{2 N}}$, where $N$ is the number of neighbor positions in the current annulus. Gray shading represents \pm 5 standard deviations.

For a given distance, both measures of anisotropy around stationary locusts (left) are small - 

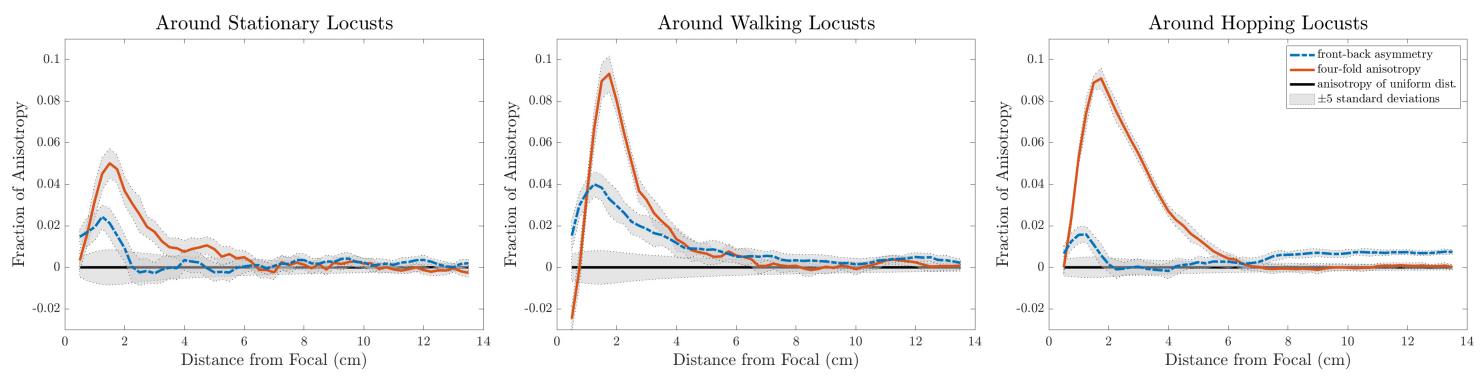

Figure 5: Anisotropy around stationary (left), walking (center), and hopping (right) locusts as functions of distance from the representative focal individual. Each plot shows front-back asymmetry $-M_{1}^{s}$ (dashed blue), four-fold anisotropy $M_{2}^{c}$ (solid orange), and anisotropy computed analytically for a uniform distribution $-M_{1}^{s}=M_{2}^{c}=0$ (black). Gray shading represents \pm 5 standard deviations. Both measures of anisotropy around stationary locusts are approximately half of the same measure around moving locusts and decrease quickly towards uniformity. Front-back asymmetry (dashed blue) peaks before decreasing slowly around walking locusts, while it is generally smaller around hopping locusts. Four-fold anisotropy (solid orange) around both walking locusts and hopping locusts peaks at a radius of $2 \mathrm{~cm}$, then decreases quickly around walking locusts and slowly around hopping locusts. At distances above $8 \mathrm{~cm}$, there is small but significant front-back asymmetry around hopping locusts. Values for distances smaller than $0.5 \mathrm{~cm}$ are computed from relatively few neighbors, resulting in the initial negative value of $M_{2}^{c}$ around walking locusts.

approximately half - compared to the same around moving locusts (center, right). Front-back asymmetry $-M_{1}^{s}$ (dashed blue), is more than twice as large around walking locusts (center) than around either other motion state. Smaller front-back asymmetry around hopping locusts at short distances may be attributed to their high movement speeds. Specifically, any neighbors or open spaces ahead of them will be behind them a fraction of a second later; our time-aggregated plots manifest this as front-back symmetry around hopping locusts. Four-fold anisotropy $M_{2}^{c}$ (solid orange) peaks around both walking locusts and hopping locusts (right) at a radius of approximately $2 \mathrm{~cm}$, but decreases quickly around walking locusts and more slowly around hopping locusts. Both anisotropy quantities decrease to near 0 at a distance between $6-8 \mathrm{~cm}$ for all motion states. Around hopping locusts, we notice a small increase in front-back asymmetry for distances greater than $8 \mathrm{~cm}$. These computations quantify our visual observations of Figure 4. Moreover, they reveal lengthscales where the anisotropies are strongest.

\section{Discussion}

Insight into animal collective behavior develops from the feedback between theoretical studies of universal models and empirical studies of the animals themselves. The modeling approach seeks to reproduce a range of incredible structures, patterns, and group strategies based on simple and often local interactions between individuals. Meanwhile, the empirical work aims to uncover the specifics of individual-level behaviors. One challenge of conducting empirical studies has been to capture individual behavior in the midst of large and often dense groups of animals. This has been a particular difficulty for locusts.

One complication is that locusts do not frequently swarm. A series of environmental conditions must align before population densities increase to levels that trigger expression of the gregarious phase and bands of marching hoppers form [54]. While diurnal rhythms might cause starlings to murmur or fish to school, locust bands appear only every few years for most species. A second obstacle is that locust's small size and mass requires high-resolution imaging. Spatial resolution is required to distinguish individuals and a high frame rate is necessary to capture extremely fast changes in direction and speed. A third difficulty is the disorganization of locust behavior, as compared with larger animals. For instance, watching surf scoters, Melanitta perspicillata, one can 
immediately observe a characteristic spacing between individual ducks as they move in an almost crystalline lattice [40]. Meanwhile, the locusts in our footage (see Sample video clips for a sample) appear to stop, walk, and hop almost at random. Identifying coherent signal amidst this inherent noise necessitates a quantity of data orders of magnitude larger than similar studies of other species.

It is, therefore, understandable that nearly all empirical studies of locusts on the individual level have been conducted in the laboratory with a relatively small number of insects. Most field studies quantify the structure and motion of locust bands only on the collective scale. The only data set providing an exception, analyzed by Buhl et al. [14, 13], relied on manual detection in one frame per second and so could not capture the movement of individuals. By contrast, our data reflect almost 20000 individual locust trajectories, resolved at 25 frames per second, allowing classification of locust motion at an unprecedentedly high resolution.

The fine timescale of our trajectory data allowed the first analysis of individual locust motion in the field. We classified motion into three distinct modes: stationary, walking, and hopping. Intermittent motion was previously quantified in laboratory studies $[9,2]$, typically as a binary between "stop" and "go" and for another species, the desert locust. Our evidence suggests a significant difference between the two moving states (walking and hopping) for the Australian plague locust. In theoretical models, intermittent motion provides a collective mechanism for density regulation $[21,12,19,11]$. By including hopping in future models, our findings may help to reproduce the dense fronts that occur in hopper bands of the Australian plague locust.

Our data has revealed novel anisotropies in the positions of neighbors around focal locusts in a natural setting. In particular, we find a lower likelihood of frontal neighbors around moving (walking and hopping) focal locusts. This represents a significant advance over the only other study that examined locust interactions in a hopper band [13]. Relying on manual data collection, that study computed a rotationally symmetric distribution from less than 20000 neighbor positions in a disc of radius greater than $28 \mathrm{~cm}$. Automated particle tracking enabled us to collect nearly 20000000 neighbor positions in a disc of radius $14 \mathrm{~cm}$. After accounting for the difference in area, this represents an increase in number of data points by a factor of $2.5 \times 10^{4}$. In turn, this revealed the previously invisible anisotropies now clearly apparent in Figures 4.

These anisotropies have not been produced by models of locust interaction and hopper band collective motion. Previous models of locust interaction are either isotropic [19, 3] or based on the idea of cannibalistic escape-and-pursuit [48, 8]. As shown explicitly by Buhl et al. [13], the escapeand-pursuit paradigm generates an anisotropy in neighbor density where localized high-density areas appear in front and behind the focal locust. This contrasts with the anisotropy found in our analysis. Models with isotropic interaction produce isotropic neighbor densities, also contrasting with our findings. New mechanisms of locust interaction are likely necessary to emulate our findings.

Anisotropies in other species Most quantitative studies of animal groups reveal structure in the position of nearby neighbors. In contrast to our findings for locusts, higher densities are observed ahead and behind the focal individual in the Serengeti wildebeest [58], surface-swimming surf scoters [40], and some species of fish [38]. This type of anisotropy is typically associated with following behavior. Various species of fish display a range of likely relative neighbor positions, including at a diagonal or with no preference [42] and laterally [41, 29]. Starlings keep their nearest neighbors on their left and right, but this preference fades when considering all neighbors in a given radius [5].

We found an area of lower density ahead of moving focal locusts, see Figure 4 (center, right), which is a unique feature when compared with other species. Quantifying this in the front-back asymmetry, Figure 5 (dashed blue curve), we established its presence at distances less than $7 \mathrm{~cm}$ around walking locusts and at distances less than $2 \mathrm{~cm}$ and greater than $7 \mathrm{~cm}$ for hopping locusts. Simultaneously, we found that distributions of neighbors around moving locusts exhibit a four-fold anisotropy similar to that of starlings, with lateral areas of high density, see Figures 4, 5 (solid orange curve). Various explanations have been given for the higher occurrence of lateral neighbors for airborne birds and some fish. As suggested by Ballerini et al. [6], this four-fold anisotropy could be due to a hydro/aerodynamic advantage, anisotropic vision, or a mechanism to avoid collisions in a high-speed group. Since these juvenile locusts do not fly, they would derive no significant aerodynamic benefit. Since locusts have essentially $360^{\circ}$ vision [47], limited sight lines are not a 
likely explanation. Given the additional presence of front-back asymmetry around moving locusts, we suggest that the mechanism causing the anisotropies revealed here is one of collision avoidance.

Collision avoidance in locusts Collision avoidance behaviors are well-documented through laboratory experiments in adult locusts; see a review by Fotowat and Gabbiani [25]. Both neural and behavioral responses occur from visual looming stimuli $[46,28]$ and have been particularly associated with collision trajectories [37, 45]. Stimuli in these studies had an area of at least $5 \mathrm{~cm} \times 5 \mathrm{~cm}$ and responses were associated to a particular size of retinal image on the eye of the locust [46]. Response behaviors have included avoidant gliding for tethered flying locusts [49] and jumping away an incoming stimulus for locusts on the ground [50]. This body of work establishes that adult locusts have the physiology necessary to sense nearby obstacles and react to avoid collisions. Our empirical findings suggest that juvenile locusts may exhibit similar visual sensing and motion adjustment; in our case in the midst of a naturally-occurring swarm, albeit while marching en mass on the ground rather than in flight. It is unclear whether this behavior may be due to the same neural circuits well-studied in adult locusts, opening up new behavioral and neurological questions.

Recommendations for locust models of collective motion A common aim of many models of locust hopper bands is to develop a universal framework that can reproduce the wide range of observed patterns from columnar structures to dense fronts. Our results reveal state-dependent elements of locust motion and interaction during marching that are yet untested by models and provide promising explanations for collective structure.

To start, we suggest a three-state model for capturing locust motion. The high level of accuracy (estimated at $85.0 \%$ ) of our method for classifying motion states supports this framework. Moreover, each motion state comprised a significant fraction (nearly 25\%) of the data derived from locusts naturally marching in the field. This makes it difficult to justify omitting any one motion state from a realistic model. Additional evidence comes from the clean division of our distribution of individual speeds shown in Figure 3. A possible implementation could use a discrete time Markov process to dictate switches between stationary, walking, and hopping states. Going further, our distributions of speeds for each state could add biologically-realistic individual variation to such a model.

Secondly, we suggest modeling locust interactions with mechanisms for collision avoidance. By contrast, existing models of locust interaction either treat equidistant neighbors the same (i.e. are isotropic) or implement the escape-and-pursuit paradigm [48, 7, 8, 31] (where motion is driven by chasing behavior, often associated with cannibalism). As noted above, we find that moving locusts (both walking and hopping) have a high-density of neighbors on the left and right. A first possible mechanism for this anisotropy might be to implement a preference for lateral neighbors. However, this alone would likely not explain the additional front-back anisotropy we observe around moving locusts.

The area of lower neighbor density immediately in front of walking locusts (Figure 4, center) suggests that when walking locusts see another individual in front of them, they react to avoid a direct collision. Perhaps they come to an abrupt stop, which could be encoded in a model by constructing a probability to switch from walking to stationary that depends on frontal neighbors. An alternative is that they might adjust the direction of their motion, in effect moving the frontal neighbor to their side. This behavior would appear in a model by using some anisotropic kernel function when updating heading direction according to nearby neighbors. In either case, a lengthscale for the behavior may be drawn from Figure 5 (center).

The anisotropy around hopping locusts (Figure 4, right) suggests a slightly different mechanism. Indeed, juvenile hoppers cannot change their motion mid-jump. Noting that the low-density area ahead of hopping locusts is narrower and more elongated than ahead of walking locusts, we suggest that one possibility is that locusts check a long and narrow area ahead of them before hopping. Then, they are more likely to hop when the path ahead is clear. Supporting this suggesection, there is a slight increase in front-back anisotropy for distances greater than $7 \mathrm{~cm}$ ahead of hopping locusts, see Figure 5 (right). This lengthscale also provides modelers with a valuable parameter when implementing such a mechanism, which could be encoded into a front-neighbor-dependent probability to switch out of the hopping state. 
We hope that future models of collective motion in locusts will incorporate some of these specifics in order to determine their effect on the collective structure and function of the hopper band. Such predictions at the band level can then be tested in future field studies.

Further Refinements There are two limitations of our study that bear acknowledging and provide opportunity for further investigation. The first is that our particle tracking implementation only identifies locust position. Consequently, we must infer heading direction from velocity. Since locusts move forward, almost never backwards or sideways, we are confident in the assumption that body orientation is equivalent to heading direction, i.e. direction of motion. The remaining issue is that stationary locusts do not have a well-defined heading direction. Via smoothing and interpolation we assigned a heading direction where we could confidently do so, but it could be preferable to apply a particle tracking software that collects body orientation as well as position.

Secondly, we employed a supervised classification method for determining motion state. While we achieved a high degree of accuracy, a classification that makes use of unsupervised learning might uncover additional motion states that are not immediately apparent to the human eye while watching the recorded footage. For instance, only after watching footage in detail did we begin to notice non-moving locusts rotating their body's orientation without advancing in any direction. This often occurred after a locust made an especially large jump. Particularly for small hoppers, these large jumps ended with a crash landing so that the insect's orientation was no longer aligned with the prevailing direction of the band's motion. The locust would rotate to align itself with passing neighbors before hopping again. Especially in conjunction with tracking software that records body orientation, identifying this behavior as distinct from other hopping might provide even stronger evidence of collision avoidance or novel patterns of interaction.

Towards predictive modeling In addition to informing theoretical models of collective behaviour, our empirical investigation of individual mechanisms contributes to the development of a well parametrized predictive locust movement model. Models of hopper band movement continue to advance towards the eventual goal of predicting the direction and distance that a given band will travel. The aim of such predictive models is to inform efficient control strategies for agricultural industry and government management agencies, possibly in real time. Predicting the likely trajectory and collective momentum of a threatening band can aid in conducting efficient surveys or implementing direct control strategies, such as pesticide barrier spraying [32]. With the addition of our findings, we are ever closer to predicting collective band behavior from evidence-based mechanisms for individual motion and interaction.

\section{Methods}

\subsection{Recording hopper bands}

We recorded video footage of eight distinct hopper bands of Australian plague locust (APL), Chortoicetes terminifera, during 3-10 of November 2010 near Hillston, New South Wales. The Australian Plague Locust Commission directed us to the area and put us in contact with the local control agency in Hillston, who then took us to potential study sites. Site locations were S33.54733 E145.06678 (for bands 1-3) and S33.20745 E145.09763 (band 4-8). The hopper bands were composed of late-instar juveniles (3rd to 5 th). Of the eight bands recorded, we analyzed four for this study.

Our recording procedure was similar to that described by Buhl et al. [14]. We mounted a camera on a tripod so that it pointed vertically downwards, with a view angle approximately perpendicular to the ground. We extended the tripod's central column so that its legs did not obstruct the field of view. This resulted in a recorded area of the ground approximately $0.6 \mathrm{~m}^{2}$ using a Panasonic camcorder which recorded in 1080i.

For most recordings, we placed the tripod in the center of a marching band. Placing the tripod often caused a temporary disturbance, which we allowed to dissipate before beginning recording once the natural flow of marching had resumed. In one case, for band 1, when the location of the 
hopper band was known and accessible, we set up the tripod ahead of the band allowing for a full recording of the center of the band from front to back. For consistency, in this case we analyzed footage from after the front had passed the camera.

For each band, we chose the recording area to be flat and devoid of vegetation. We used areas located away from major obstacles that could prevent or impede locust marching such as trees, creeks, and patches of dense vegetation. We placed a sheet of plywood in the camera's view frame to provide a uniform background. We recorded the scale by placing a ruler in the field of view at the beginning of the video, or else by the known dimensions of the plywood $(120 \mathrm{~cm} \times 60 \mathrm{~cm})$.

For the purposes of this study, we selected four recordings where we observe sustained marching. Each video has a resolution of $1920 \times 1080$ pixels and consists of 25 interlaced frames per second. For this study, a total of twenty-seven minutes of footage was analyzed, representing 24300 frames. For a sample of our video footage, see Sample video clips.

\subsection{Extracting numerical trajectories (via motion tracking with Track- Mate)}

We analyzed the footage using particle-tracking software TrackMate [57], a plugin for ImageJ. This software includes a suite of established particle detection and trajectory-linking algorithms, along with a user-friendly GUI and interoperability with Matlab for scripted batch tracking. For more on particle-tracking software and to see how an early version of TrackMate performed, see [15].

Video preprocessing Before tracking, we preprocessed the videos using ffmpeg [24] and Fiji [51] (ImageJ). Using ffmpeg we clipped the videos into computationally manageable lengths of 1 min. These clips were deinterlaced and saved to a nv12 AVI file format, which can be imported into ImageJ. Next, we loaded these clips into Fiji, a distribution of ImageJ that ships with the TrackMate plugin. In Fiji, we further processed the clips by applying a grayscale filter, inverting the colors, subtracting the median image (which effectively removes the nonmoving background), adjusting the brightness and contrast, and blurring (which smooths over remaining dirt particles and makes locust images more circular). This processing procedure reduced background noise in the video and prepared it for TrackMate, which performs best on approximately circular bright particles against a dark background.

Tracking algorithms and parameters A full list of the tracking parameters we used appears in Table 1; here we discuss the two major algorithms. The first step in particle tracking is to examine each frame and detect the location of particles (called "spots" in TrackMate). We used TrackMate's Laplacian of Gaussians for our spot detection algorithm because it performs best for spots with diameter 5-20 pixels, which includes our blurred locusts. Mathematically, a Gaussian filter with standard deviation tuned by the expected spot radius is applied to the image. This smoothed image is then processed with a discrete Laplacian operator that detects local maxima. After spots have been detected in each frame, they are linked into trajectories (called "tracks"). TrackMate's Linear Motion Tracker links spots assuming a roughly constant velocity between frames, which fits our video well because a typical locust reaction time of $0.03 \mathrm{~s}$ [26] is close to the time between our frames $(0.04 \mathrm{~s})$. To link spots between a pair of frames, this algorithm combines a cost-based assignment problem developed by Jaqaman et al. [36] with predictions from a Kalman filter for each locust. More technical details can be found in the TrackMate documentation [56].

Evaluating accuracy TrackMate was also used for manual tracking to create two 10-s data sets, which we treat as our ground truth. These data include a total of 542 trajectories composed of 68308 locust positions. We used the first data set to tune various parameters and the second to evaluate the accuracy of our data. We henceforth refer to the first as our training data and to the second as our test data.

To compare a ground-truth data set to its analogous data obtained from automatic tracking, we use similarity coefficients adapted from the Jacard Similarity Coefficient used in a challenge from 


\begin{tabular}{rlc}
\hline TrackMate Parameter & Description & Value(s) \\
\hline Spot Detector & the algorithm used to detect spots (particles) in each frame & Laplacian of Gaussians (LoG) \\
Estimated blob diameter & the algorithm's first estimate for the size of each spot, tunes the Gaussian filter & $0.52 \mathrm{~cm}$ \\
Threshold & the minimum Quality* value required to detect a spot & 0.1 \\
Initial thresholding & the minimum Quality* value required to keep a spot before linking & $0.95-2.3$ \\
\hline Tracker & the algorithm used to link spots into tracks (trajectories) between frames & Linear motion LAP tracker \\
Initial search radius & maximal distance allowed when linking two spots to create a new track & $2.02 \mathrm{~cm}$ \\
Search radius & maximal distance allowed when linking two spots to continue a track & $4.31 \mathrm{~cm}$ \\
Max frame gap & maximal number of frames allowed between two spots when continuing a track & $1 \mathrm{frame}$ \\
Min displacement & minimal distance required to keep a track, eliminates stationary dirt & $8 \mathrm{~cm}$ \\
\hline
\end{tabular}

Table 1: Tracking parameters for TrackMate, descriptions, and values used in our tracking. *Quality is defined as the local maximum value when the LoG filter is applied, see [57, Section 5.1.2] for details.

the International Symposium on Biomedical Imaging [15]. For spot similarity we compute the Spot Similarity Coefficient

$$
\mathrm{SSC}=\frac{\mathrm{TP}}{\mathrm{TP}+\mathrm{FP}+\mathrm{FN}}
$$

where TP is the number of true positive detections, FP is the number of false positives, and FN is the number of false negatives. Each of these is computed by an assignment problem between the two corresponding frames of each data set and a maximal assignment distance of $0.75 \mathrm{~cm}$, less than $\frac{1}{2}$ of a locust body length. The Link Similarity Coefficient is computed in a similar manner where we take pairs of frames from each data set. Each of these values ranges between 0 (no true positives) to 1 (perfect tracking). By tuning our tracking parameters we optimized the accuracy, achieving $\mathrm{SSC}=0.97$ and $\mathrm{LSC}=0.96$ for the training data. Evaluating these parameters against our test data we measured a spot accuracy of SSC $=0.91$ and link accuracy of LSC $=0.90$. This represents accurate tracking in a preponderance of the data. Given that we analyze 3369723 data points, this should be more than sufficient to distinguish signal from inherent noise. See Sample video clips for a sample video illustrating the accuracy of our tracking.

\subsection{Inferring motion}

From the trajectory data for each locust, we inferred instantaneous velocity and decomposed it into speed and heading direction. Having such small mass, locusts can exhibit extremely large accelerations and we observed that locusts often changed their motion between frames of our video, which are separated by $0.04 \mathrm{~s}$. Thus, more accurate velocity values can be obtained by smoothing the position data [52]. In order to choose an optimal interval length for the smoothing filter, we measured the timescale of significant autocorrelation in a manner similar to Ying et al. [61]. For a given trajectory, we computed the time series of distances traveled between frames and tested the null hypothesis that a unit root is present with an Augmented Dickey-Fuller (ADF) test. Of our ground-truth data, we found that $93.3 \%$ of time series successfully rejected the null hypothesis and are therefore statistically stationary. We next computed the autocorrelation function with up to 20 time lags for each time in the trajectory. We judged a given time lag not to be significantly correlated with the original time if its autocorrelation function was less than two standard deviations from 0.

We recorded the time lag at the beginning of the first period of insignificantly correlated time lags. This represents the timescale after which a locust's trajectory is not significantly correlated with itself. Across our ground truth data, we found a median correlation time of 3 frames (SD 4.8), or $0.12 \mathrm{~s}(\mathrm{SD} 0.19 \mathrm{~s})$. We therefore selected a weighted Gaussian filter over 8 frames as our smoothing function, which effectively links a given position only to 4 frames, or $0.16 \mathrm{~s}$, in the future and four frames in the past. 
After smoothing the position data, we computed velocities using a central difference method. Speed was calculated as the magnitude of the velocity. Taking the angle of the velocity in a standard coordinate system ( $x$-axis to the right, $y$-axis up) we calculated heading direction. Note that heading direction is not well-defined for an unmoving locust. In fact, small fluctuations in position (inherited from automatic tracking) can produce large fluctuations in the heading direction. To account for this, we used linear interpolation to recompute the heading direction for stationary locusts after we classified their motion states.

\subsection{Classifying motion state}

Watching the raw footage, we observed locusts moving in one of three distinct motion states:

1. Stationary - locusts do not advance in any direction, may rotate their body's orientation

2. Walking - locusts advance relatively slowly without leaving the ground, with short pauses separated by $\sim 1$ s of motion

3. Hopping - locusts advance quickly through erratic jumps, sometimes with pauses of up to $\sim 1 \mathrm{~s}$ between jumps.

Distinct motion states have been previously recognized in locusts, including all three of these [22, $59,2]$. We manually classified all locusts in the two manually-tracked 10 s clips, creating our training and test data for automatic classification.

We implemented a support vector machine (SVM) to automatically classify each locust in each frame into one of the three motion states. SVMs are popular classification tools from machine learning - see the book by James et al. [34, Ch. 9] for an accessible introduction. SVMs compute an optimal boundary between each class of data points in a training data set, then use those boundaries to classify the remaining data. The boundaries depend only on the data points closest to them, called the support vectors, so the method is not sensitive to variations in well classified data points. For our application, we used a nonlinear boundary via a Gaussian kernel function. Computing the boundary amounts to an optimization problem where misclassified data points are assigned a penalty value based on their distance from the boundary in a higher-dimensional space. We implemented our SVM using Matlab's fitcecoc() function.

After initial exploration of the data, we chose the following trajectory features at each time to classify locust motion states in that frame: first, instantaneous speed (after position smoothing, described above) distinguishes locusts that are stationary at the current time; second, the magnitude of average velocity distinguishes a stationary locust from one that has paused between jumps; third, the standard deviation of speed distinguishes between hopping (fast and intermittent speed) and walking (slow and almost constant speed); fourth, the minimum of the forward and backward maximums helps to discern between stationary and hopping locusts near the beginning or end of their trajectories. For the latter three we used a time window of 15 frames, equivalent to $0.6 \mathrm{~s}$. We chose this number of frames by tuning the classification accuracy of our SVM on the training data. To avoid overfitting, we optimized various internal hyperparameters of our SVM using cross-validation on the training data. We trained the SVM using the same data set and found that it correctly classified $86.8 \%$ of the training data. We then evaluated the accuracy of our SVM on the second ground-truth data set and found correct classification for $85.0 \%$ of the test data. Given the magnitude of the whole data set, this represents a level of accuracy that should easily distinguish trends from noise. See Sample video clips for a sample video that illustrates the accuracy of our motion classification.

\subsection{Measuring collective marching}

Since we wish to analyze the behavior of individuals during marching, we compute three quantities that are associated with this collective behavior. In each frame of video, we compute the density $D$ by counting the number of locusts detected and dividing by the physical area in the frame. This is a relatively coarse measure of density since even the most highly populated frames do not approach the 
maximal packing density, but it is nonetheless consistent with previous measurements. Typically, a density greater than 20 locusts $/ \mathrm{m}^{2}$ has been associated with marching [55, 12]. We also compute the polarization $P$ as the length of the average of the direction vectors $\left\langle\cos \phi_{i}, \sin \phi_{i}\right\rangle$, where $\phi_{i}$ is the heading direction of the $i^{\text {th }}$ locust. For a frame of video containing $N$ locusts, these quantities are

$$
D=\frac{N}{\text { area }} \quad \text { and } \quad P=\frac{1}{N}\left\|\sum_{i=1}^{N}\left\langle\cos \phi_{i}, \sin \phi_{i}\right\rangle\right\| .
$$

Polarization is a commonly-used order parameter ranging from 0 (completely disordered) to 1 (completely aligned). Marching locusts have demonstrated polarization values between 0.6 and 0.9 [14]. Polarization is known to be an ambiguous measure of alignment, since low values can result from either disorder or from two groups aligned in opposite directions. To supplement polarization, we also compute an index that captures an adaptation of Boltzmann entropy.

This entropy index $E$ varies from 0 (completely aligned) to 1 (completely disordered) and was originally described by Baldassarre [4]. For marching locusts, the same entropy index has been reported between 0.75 and 0.9 [14] and we follow the same implementation therein. For a frame with $N$ locusts, we distribute their heading directions into an arbitrary number $C=72$ of bins with width $2 \pi / C$. Letting $N_{i}$ be the number of angles in the $i^{\text {th }}$ bin, we compute the entropy index as

$$
E=\frac{1}{\ln w^{\max }} \ln \left(\frac{N !}{N_{1} ! N_{2} ! \cdots N_{C} !}\right)=\frac{1}{\ln w^{\max }}\left(\ln (N !)-\sum_{i=1}^{C} \ln \left(N_{i} !\right)\right)
$$

where $w^{\max }$ is the maximal value of $\frac{N !}{N_{1} ! N_{2} ! \cdots N_{C} !}$ among all possible distributions of $N$ heading directions into $C$ classes, i.e. a nearly uniform distribution where each $N_{i}$ is either $\left\lfloor\frac{N}{C}\right\rfloor$ or $\left\lfloor\frac{N}{C}\right\rfloor+1$. For $N>170$, we use Stirling's approximation $\ln (N !) \approx N \ln N-N+\frac{1}{2} \ln (2 \pi N)$ to avoid computational issues with large factorials. We acknowledge that the number of bins $C$ can have a significant effect on the index. For a fixed $C$, however, the index $E$ provides a stable relative measure of order. We use the same number of bins as Buhl et al. [14] because our goal is a direct comparison that establishes ordered marching for the locusts in our study.

\subsection{Quantifying anisotropy in neighbor density}

Using the position and heading direction data, we aggregated relative positions of neighbors into density distributions using a methodology similar to Buhl et al. [13]. For each focal locust in a frame, we computed the relative position of each neighbor as its position in a coordinate frame with the focal individual at the origin, the $y$-axis pointing from tail to head, and the $x$-axis protruding to its right. We avoid biases introduced by the edges of the frame by taking two precautions. First, we only considered neighbors within $14 \mathrm{~cm}$ of the focal locust. This distance includes the only estimate we know of for the interaction range between locusts, which is $13.5 \mathrm{~cm}$. Second, we used the Hanisch correction [30, 18], which ignores any neighbor at a distance further from the focal individual than the nearest edge. We discretized this distribution of relative neighbor positions into square bins with side length $\Delta x=\Delta y=0.5 \mathrm{~cm}$. We normalized the counts in each bin by the average density over all focal locusts divided by the average density in the whole area. The effect of this normalization factor is that as the distribution approaches homogeneity, the value in each bin approaches 1 . We call these distributions relative neighbor densities and plot them as two-dimensional maps where color indicates density. This relative density can equivalently be thought of as the likelihood of finding a neighbor in a given position relative to a focal individual.

We also extracted the angle of each relative neighbor position and examined these as a distribution on the circle. An isotropic distribution of neighbors would correspond to a uniform distribution of their angles on the circle, so we used the Hodges-Ajne test for uniformity as described and implemented in the Matlab Circular Statistics Toolbox [10]. We quantify the non-uniformity of these distributions using trigonometric moments as described by Jammalamadaka and SenGupta 
[35]. Given a set of $N$ angles $\phi_{i}$, the $p^{\text {th }}$ trigonometric moments are

$$
M_{p}^{s}=\frac{1}{N} \sum_{i=1}^{N} \sin \left(p \phi_{i}\right) \quad \text { and } \quad M_{p}^{c}=\frac{1}{N} \sum_{i=1}^{N} \cos \left(p \phi_{i}\right) .
$$

Mathematically, these are discretizations of the coefficients in the Fourier series expansion of a density function on the circle. In this way, they capture how much the data is concentrated into $p$ groups, i.e. the Fourier modes on the circle. Physically, the vector $\mathbf{M}_{p}=\left\langle M_{p}^{c}, M_{p}^{s}\right\rangle$ has magnitude $\left|\mathbf{M}_{p}\right|=\sqrt{\left(M_{p}^{c}\right)^{2}+\left(M_{p}^{s}\right)^{2}}$, which provides a dimensionless measure that quantifies how much the data is concentrated into $p$ equally-spaced groups. The vector $\mathbf{M}_{p}$ points in the direction $p \phi_{p}$ where $\phi_{p}$ is the angle of one of the $p$ concentrations of data. A uniform distribution on the circle has $\left|\mathbf{M}_{p}\right|=0$ and a completely concentrated distribution where all $\phi_{i}$ 's are equal has $\left|\mathbf{M}_{p}\right|=1$.

We are particularly interested in $-M_{1}^{s}$ and $M_{2}^{c}$. Recall the coordinate system in our relative neighbor density plots, see Figure 1 (right) and Figure 4. For the focal locust in the center and facing upwards, $-M_{1}^{s}$ quantifies the front-back asymmetry. With the negative sign, this quantity is larger when there is a lower density in front and a higher density behind the focal locust. It is relatively stable to fluctuations in density on the left and right. By contrast, $M_{2}^{c}$ quantifies the four-fold anisotropy around the focal locust. It is largest when there are high-density concentrations to the left and right and lower-density areas in front and behind. In Figure 5, we examine how these quantities vary by distance from the focal individual by looking at subsets of neighbors in concentric annuli.

\section{Acknowledgements}

We thank the Australian Plague Locust Commission, the New South Wales Department of Primary Industries, Darron Cullen, Katie Robinson and Feng Hu for their help in the field. We thank Michael Culshaw-Maurer and Christopher Strickland for valuable conversations on how best to handle and analyze the large quanity of data. JW thanks Andrew Bernoff for support and guidance from the conception of the analysis to its conclusion. JL thanks Suzanne Amador Kane for her insight and mentorship throughout a preliminary version of the data investigation. JW was supported by an NSF Postdoctoral Fellowship Grant DMS-1902818. JL was supported by the HMC Data Science REU, NSF Grant-1757952. AK, SL, and TS were supported by the Undergraduate Research Opportunities program at Harvey Mudd College. SJS, GAS and JB were funded by an ARC Linkage LP150100479. JB was funded by an ARC Future Fellowship FT110100082. 


\section{References}

[1] Gil Ariel and Amir Ayali. Locust Collective Motion and Its Modeling. PLOS Computational Biology, pages 1-25, 2015. doi: 10.1371/journal.pcbi.1004522. URL http://dx.doi.org/10.1371/journal. pcbi. 1004522 .

[2] Gil Ariel, Yotam Ophir, Sagi Levi, Eshel Ben-Jacob, and Amir Ayali. Individual pause-and-go motion is instrumental to the formation and maintenance of swarms of marching locust nymphs. PLOS One, 9(7):e101636, 2014.

[3] A. Bach. Exploring locust hopper bands emergent patterns using parallel computing. Master's thesis, Université Paul Sabatier Toulouse III, June 2018.

[4] Gianluca Baldassarre. Self-organization as phase transition in decentralized groups of robots: A study based on boltzmann entropy. In Advanced Information and Knowledge Processing, pages 157177. Springer London, 2013. doi: 10.1007/978-1-4471-5113-5_7. URL https://doi.org/10.1007\% 2F978-1-4471-5113-5_7.

[5] M. Ballerini, N. Cabibbo, R. Candelier, A. Cavagna, E. Cisbani, I. Giardina, V. Lecomte, A. Orlandi, G. Parisi, A. Procaccini, M. Viale, and V. Zdravkovic. Interaction ruling animal collective behavior depends on topological rather than metric distance: Evidence from a field study. Proceedings of the National Academy of Sciences, 105(4):1232-1237, 2008. ISSN 0027-8424. doi: 10.1073/pnas.0711437105. URL https://www . pnas .org/content/105/4/1232.

[6] Michele Ballerini, Nicola Cabibbo, Raphael Candelier, Andrea Cavagna, Evaristo Cisbani, Irene Giardina, Alberto Orlandi, Giorgio Parisi, Andrea Procaccini, Massimiliano Viale, and Vladimir Zdravkovic. Empirical investigation of starling flocks: a benchmark study in collective animal behaviour. Animal Behaviour, 76(1):201-215, jul 2008. doi: 10.1016/j.anbehav.2008.02.004. URL https ://doi.org/10.1016\%2Fj . anbehav.2008.02.004.

[7] Sepideh Bazazi, Jerome Buhl, Joseph J. Hale, Michael L. Anstey, Gregory A. Sword, Stephen J. Simpson, and Iain D. Couzin. Collective motion and cannibalism in locust migratory bands. Current Biology, 18(10):735-739, 2019/06/26 2008. doi: 10.1016/j.cub.2008.04.035. URL https://doi.org/10.1016/j . cub.2008.04.035.

[8] Sepideh Bazazi, Pawel Romanczuk, Sian Thomas, Lutz Schimansky-Geier, Joseph J. Hale, Gabriel A. Miller, Gregory A. Sword, Stephen J. Simpson, and Iain D. Couzin. Nutritional state and collective motion: from individuals to mass migration. Proc. R. Soc. B, 278(1704):356-363, aug 2010. doi: 10.1098/rspb.2010.1447. URL https://doi.org/10.1098\%2Frspb. 2010.1447.

[9] Sepideh Bazazi, Frederic Bartumeus, Joseph J. Hale, and Iain D. Couzin. Intermittent motion in desert locusts: Behavioural complexity in simple environments. PLoS Computational Biology, 8(5):e1002498, May 2012. ISSN 1553-7358. doi: 10.1371/journal.pcbi.1002498. URL http://dx.doi.org/10.1371/ journal.pcbi.1002498.

[10] Philipp Berens. CircStat: A MATLAB Toolbox for circular statistics. Journal of Statistical Software, 31(10), 2009. doi: 10.18637/jss.v031.i10. URL https://doi.org/10.18637\%2Fjss.v031.i10.

[11] Andrew J. Bernoff, Michael Culshaw-Maurer, Rebecca A. Everett, Maryann E. Hohn, W. Christopher Strickland, and Jasper Weinburd. Agent-based and continuous models of hopper bands for the australian plague locust: How resource consumption mediates pulse formation and geometry. PLOS Computational Biology, 16(5):e1007820, may 2020. doi: 10.1371/journal.pcbi.1007820. URL https://doi.org/10. 1371\%2Fjournal.pcbi. 1007820.

[12] J. Buhl, D.J.T. Sumpter, I. D. Couzin, J.J. Hale, E. Despland, E.R. Miller, and S. J. Simpson. From Disorder to Order in Marching Locusts. Science, 312:1402-1406, 2006. doi: 10.1126/science.1125142.

[13] J Buhl, Gregory A Sword, and Stephen J Simpson. Using field data to test locust migratory band collective movement models. Interface Focus, 2:757-763, 2012.

[14] Jerome Buhl, Gregory A Sword, Fiona J Clissold, and Stephen J Simpson. Group structure in locust migratory bands. Behavioral Ecology and Sociobiology, 65(2):265-273, 2011. 
[15] Nicolas Chenouard, Ihor Smal, Fabrice de Chaumont, Martin Maška, Ivo F. Sbalzarini, Yuanhao Gong, Janick Cardinale, Craig Carthel, Stefano Coraluppi, Mark Winter, Andrew R. Cohen, William J. Godinez, Karl Rohr, Yannis Kalaidzidis, Liang Liang, James Duncan, Hongying Shen, Yingke Xu, Klas E. G. Magnusson, Joakim Jaldén, Helen M. Blau, Perrine Paul-Gilloteaux, Philippe Roudot, Charles Kervrann, François Waharte, Jean-Yves Tinevez, Spencer L. Shorte, Joost Willemse, Katherine Celler, Gilles P. van Wezel, Han-Wei Dan, Yuh-Show Tsai, Carlos Ortiz de Solórzano, Jean-Christophe Olivo-Marin, and Erik Meijering. Objective comparison of particle tracking methods. Nature Methods, 11(3):281-289, Mar 2014. ISSN 1548-7105. doi: 10.1038/nmeth.2808. URL https://doi.org/10.1038/nmeth.2808.

[16] L.R. Clark. Behaviour of Swarm Hoppers of the Australian Plague locust Chortoicetes terminifera (Walker). Commonwealth Scientific and Industrial Research Organization, Australia, 245:5-26, 1949.

[17] Felipe Cucker and Steve Smale. On the mathematics of emergence. Japanese Journal of Mathematics, 2(1):197-227, mar 2007. doi: 10.1007/s11537-007-0647-x. URL https://doi.org/10.1007\% 2Fs11537-007-0647-x.

[18] Peter Diggle. Statistical analysis of spatial and spatio-temporal point patterns. CRC press, 2013.

[19] Jamila Dkhili, Uta Berger, Lalla Mina Idrissi Hassani, Saïd Ghaout, Ronny Peters, and Cyril Piou. Self-organized spatial structures of locust groups emerging from local interaction. Ecological Modelling, 361:26-40, October 2017. ISSN 03043800. doi: 10.1016/j.ecolmodel.2017.07.020.

[20] Jamila Dkhili, Koutaro Ould Maeno, Lalla Mina Idrissi Hassani, Said Ghaout, and Cyril Piou. Effects of starvation and vegetation distribution on locust collective motion. Journal of Insect Behavior, 32(3): 207-217, May 2019. ISSN 1572-8889. doi: 10.1007/s10905-019-09727-8. URL http://dx.doi.org/10. 1007/s10905-019-09727-8.

[21] Leah Edelstein-Keshet, James Watmough, and Daniel Grunbaum. Do travelling band solutions describe cohesive swarms? An investigation for migratory locusts. Journal of Mathematical Biology, 36:515-549, 1998. doi: $10.1007 / \mathrm{s} 002850050112$.

[22] Peggy Ellis and Clifford Ashall. Field studies on diurnal behaviour, movement and aggregation in the desert locust (schistocerca gregaria forsk.). Anti-Locust Bulletin, 25:4-94, 1957.

[23] R. A. Farrow. Population dynamics of the Australian plague locust, Chortoicetes terminifera (Walker), in Central Western New South Wales I. reproduction and migration in relation to weather. Australian Journal of Zoology, 27(5):681-689, 1979. ISSN 14465698. doi: 10.1071/ZO9790717.

[24] FFmpeg Developers. ffmpeg. Open Source, Accessed May Accessed 2020. URL https://ffmpeg.org/ ffmpeg.html\#Authors.

[25] Haleh Fotowat and Fabrizio Gabbiani. Collision detection as a model for sensory-motor integration. Annual Review of Neuroscience, 34(1):1-19, jul 2011. doi: 10.1146/annurev-neuro-061010-113632. URL https ://doi.org/10.1146\%2Fannurev-neuro-061010-113632.

[26] T. Friedel. The vibrational startle response of the desert locust schistocerca gregaria. Journal of Experimental Biology, 202(16):2151-2159, aug 1999. doi: 10.1242/jeb.202.16.2151. URL https://doi. org/10.1242\%2Fjeb.202.16.2151.

[27] Fillipe Georgiou, Jerome Buhl, J. E. F. Green, Bishnu Lamichhane, and Ngamta Thamwattana. Modelling locust foraging: How and why food affects group formation. PLOS Computational Biology, 17(7): e1008353, jul 2021. doi: 10.1371/journal.pcbi.1008353. URL https://doi.org/10.1371\%2Fjournal. pcbi. 1008353.

[28] John R. Gray, R. Meldrum Robertson, and Jessica K. Lee. Activity of descending contralateral movement detector neurons and collision avoidance behaviour in response to head-on visual stimuli in locusts. Journal of Comparative Physiology A, 187(2):115-129, apr 2001. doi: 10.1007/s003590100182. URL https://doi.org/10.1007\%2Fs003590100182.

[29] Daniel Grünbaum, Steven Viscido, and Julia K. Parrish. Extracting interactive control algorithms from group dynamics of schooling fish. In V. Kumar, N. Leonard, and A.S. Morse, editors, Cooperative Control. Lecture Notes in Control and Information Science, volume 309, pages 103-117. Springer Berlin Heidelberg, nov 2004. doi: 10.1007/978-3-540-31595-7_6. URL https://doi.org/10.1007\% 2F978-3-540-31595-7_6. 
[30] K.H. Hanisch. Some remarks on estimators of the distribution function of nearest neighbour distance in stationary spatial point processes. Series Statistics, 15(3):409-412, jan 1984. doi: 10.1080/ 02331888408801788. URL https://doi.org/10.1080\%2F02331888408801788.

[31] Matthew J. Hansen, Jerome Buhl, Sepideh Bazazi, Stephen J. Simpson, and Gregory A. Sword. Cannibalism in the lifeboat — collective movement in australian plague locusts. Behavioral Ecology and Sociobiology, 65(9):1715-1720, apr 2011. doi: 10.1007/s00265-011-1179-1. URL https: //doi.org/10.1007\%2Fs00265-011-1179-1.

[32] J. Holt and J.F. Cooper. A model to compare the suitability of locust hopper targets for control by insecticide barriers. Ecological Modelling, 195(3-4):273-280, jun 2006. doi: 10.1016/j.ecolmodel.2005. 11.026. URL https://doi.org/10.1016\%2Fj.ecolmodel.2005.11.026.

[33] D. M. Hunter, L. Mcculloch, and P. A. Spurgin. Aerial detection of nymphal bands of the Australian plague locust (Chortoicetes terminifera (Walker)) (Orthoptera : Acrididae). Crop Protection, 27:118123, 2008. doi: 10.1016/j.cropro.2007.04.016.

[34] Gareth James, Daniela Witten, Trevor Hastie, and Robert Tibshirani. An introduction to statistical learning, volume 112. Springer, 2013.

[35] S Rao Jammalamadaka and Ashis SenGupta. Topics in Circular Statistics. World Scientific, apr 2001. doi: 10.1142/4031. URL https://doi.org/10.1142\%2F4031.

[36] Khuloud Jaqaman, Dinah Loerke, Marcel Mettlen, Hirotaka Kuwata, Sergio Grinstein, Sandra L Schmid, and Gaudenz Danuser. Robust single-particle tracking in live-cell time-lapse sequences. Nature Methods, 5(8):695-702, jul 2008. doi: 10.1038/nmeth.1237. URL https://doi.org/10.1038\%2Fnmeth. 1237.

[37] S Judge and F Rind. The locust DCMD, a movement-detecting neurone tightly tuned to collision trajectories. Journal of Experimental Biology, 200(16):2209-2216, aug 1997. doi: 10.1242/jeb.200.16. 2209. URL https://doi.org/10.1242\%2Fjeb.200.16.2209.

[38] Yael Katz, Kolbjørn Tunstrøm, Christos C. Ioannou, Cristián Huepe, and Iain D. Couzin. Inferring the structure and dynamics of interactions in schooling fish. Proceedings of the National Academy of Sciences, 108(46):18720-18725, 2011. ISSN 0027-8424. doi: 10.1073/pnas.1107583108. URL https: //www.pnas.org/content/108/46/18720.

[39] Daniel Knebel, Ciona Sha-ked, Noa Agmon, Gil Ariel, and Amir Ayali. Collective motion as a distinct behavioral state of the individual. iScience, 24(4):102299, apr 2021. doi: 10.1016/j.isci.2021.102299. URL https://doi.org/10.1016\%2Fj.isci.2021.102299.

[40] Ryan Lukeman, Yue-Xian Li, and Leah Edelstein-Keshet. Inferring individual rules from collective behavior. Proceedings of the National Academy of Sciences, 107(28):12576-12580, 2010. ISSN 00278424. doi: 10.1073/pnas.1001763107. URL https://www.pnas.org/content/107/28/12576.

[41] Brian L. Partridge. The structure and function of fish schools. Scientific American, 246(6):114-123, 1982.

[42] Brian L. Partridge, Tony Pitcher, J. Michael Cullen, and John Wilson. The three-dimensional structure of fish schools. Behavioral Ecology and Sociobiology, 6(4):277-288, mar 1980. doi: 10.1007/bf00292770. URL https://doi.org/10.1007\%2Fbf00292770.

[43] Meir Paul Pener and Stephen J. Simpson. Locust Phase Polyphenism: An Update. In Advances in Insect Physiology, volume 36, pages 1-272. Elsevier, 2009. ISBN 978-0-12-374828-7. doi: 10.1016/ S0065-2806(08)36001-9.

[44] Craig W. Reynolds. Flocks, herds, and schools: A distributed behavioral model. Computer Graphics (SIGGRAPH '87 Conference Proceedings), 21(4):25-34, 1987.

[45] R. M. Robertson and A. G. Johnson. Collision avoidance of flying locusts: Steering torques and behavior. Journal of Experimental Biology, 183(1):35-60, oct 1993. doi: 10.1242/jeb.183.1.35. URL https://doi.org/10.1242\%2Fjeb.183.1.35. 
[46] R. M. Robertson and A. G. Johnson. Retinal image size triggers obstacle avoidance in flying locusts. Naturwissenschaften, 80(4):176-178, apr 1993. doi: 10.1007/bf01226378. URL https://doi.org/10. $1007 \% 2 \mathrm{Fbf} 01226378$.

[47] Stephen M. Rogers, George W. J. Harston, Fleur Kilburn-Toppin, Thomas Matheson, Malcolm Burrows, Fabrizio Gabbiani, and Holger G. Krapp. Spatiotemporal receptive field properties of a looming-sensitive neuron in solitarious and gregarious phases of the desert locust. J. Neurophysiol, 103(2):779-792, feb 2010. doi: 10.1152/jn.00855.2009. URL https://doi.org/10.1152\%2Fjn.00855. 2009.

[48] Pawel Romanczuk, Iain D Couzin, and Lutz Schimansky-Geier. Collective Motion due to Individual Escape and Pursuit Response. Physical Review Letters, 010602:1-4, 2009. doi: 10.1103/PhysRevLett. 102.010602.

[49] Roger D. Santer, Peter J. Simmons, and F. Claire Rind. Gliding behaviour elicited by lateral looming stimuli in flying locusts. Journal of Comparative Physiology A, 191(1):61-73, nov 2004. doi: 10.1007/ s00359-004-0572-x. URL https://doi.org/10.1007\%2Fs00359-004-0572-x.

[50] Roger D. Santer, Yoshifumi Yamawaki, F. Claire Rind, and Peter J. Simmons. Motor activity and trajectory control during escape jumping in the locust locusta migratoria. Journal of Comparative Physiology A, 191(10):965-975, jul 2005. doi: 10.1007/s00359-005-0023-3. URL https://doi.org/10. 1007\%2Fs00359-005-0023-3.

[51] Johannes Schindelin, Ignacio Arganda-Carreras, Erwin Frise, Verena Kaynig, Mark Longair, Tobias Pietzsch, Stephan Preibisch, Curtis Rueden, Stephan Saalfeld, Benjamin Schmid, Jean-Yves Tinevez, Daniel James White, Volker Hartenstein, Kevin Eliceiri, Pavel Tomancak, and Albert Cardona. Fiji: an open-source platform for biological-image analysis. Nature Methods, 9(7):676-682, jun 2012. doi: 10.1038/nmeth.2019. URL https://doi.org/10.1038\%2Fnmeth. 2019.

[52] Kenichi Shibata and Takashi Amemiya. How to decide window-sizes of smoothing methods: A goodness of fit criterion for smoothing oscillation data. IEICE Transactions on Electronics, E102.C(2): 143-146, feb 2019. doi: 10.1587/transele.2018oms0003. URL https://doi.org/10.1587\%2Ftransele. $20180 \mathrm{~ms} 0003$.

[53] David Sumpter. Collective animal Behavior. Princeton University Press, 2010.

[54] Gregory A. Sword, Michel Lecoq, and Stephen J. Simpson. Phase polyphenism and preventative locust management. Journal of Insect Physiology, 56(8):949-957, 2010. ISSN 0022-1910. doi: https: //doi.org/10.1016/j.jinsphys.2010.05.005. URL https://www.sciencedirect.com/science/article/ pii/S0022191010001460. Locust Research in the Age of Model Organisms In honor of M.P. Pener's 80th Birthday.

[55] P. M. Symmons and K. Cressman. Desert locust guidelines. Technical report, Food and Agriculture Organization of the United Nations, Rome, 2001.

[56] Jean-Yves Tinevez. TrackMate Manual, Aug 2016. URL https://imagej.net/media/plugins/ trackmate/trackmate-manual.pdf.

[57] Jean-Yves Tinevez, Nick Perry, Johannes Schindelin, Genevieve M. Hoopes, Gregory D. Reynolds, Emmanuel Laplantine, Sebastian Y. Bednarek, Spencer L. Shorte, and Kevin W. Eliceiri. Trackmate: An open and extensible platform for single-particle tracking. Methods, 115:80 - 90, 2017. ISSN 1046-2023. doi: https://doi.org/10.1016/j.ymeth.2016.09.016. URL http://www. sciencedirect.com/ science/article/pii/S1046202316303346. Image Processing for Biologists.

[58] Colin J. Torney, J. Grant C. Hopcraft, Thomas A. Morrison, Iain D. Couzin, and Simon A. Levin. From single steps to mass migration: the problem of scale in the movement ecology of the serengeti wildebeest. Phil. Trans. R. Soc. B, 373(1746):20170012, mar 2018. doi: 10.1098/rstb.2017.0012. URL https ://doi.org/10.1098\%2Frstb. 2017.0012.

[59] B. Uvarov. Grasshoppers and Locusts, volume 2. Cambridge University Press, London, UK, 1977.

[60] T. Vicsek, A. Czirok, E. Ben Jacob, I. Cohen, and O. Shochet. Novel type of phase-transition in a system of self-driven particles. Phys. Rev. Lett., 75(6):1226-1229, August 1995. 
[61] Wenxia Ying, Gabriel Huerta, Stanly Steinberg, and Martha Zúñiga. Time series analysis of particle tracking data for molecular motion on the cell membrane. Bulletin of Mathematical Biology, 71(8):1967-2024, aug 2009. doi: 10.1007/s11538-009-9434-6. URL https://doi.org/10.1007\% 2Fs11538-009-9434-6.

\section{Appendices}

\section{A Sample video clips}

Here we present links to a $10 \mathrm{~s}$ video clip from our raw video footage. We manually tracked the locusts in this clip to create the "ground-truth" test data that tuned the automatic tracking process and trained the motion classification machine.

1. Sample video footage showing juvenile Australian plague locusts, Chortoicetes terminifera, marching in a hopper band in the field. Recordings were captured by a camera mounted on a tripod with an extended arm and directed vertically down. A sheet of plywood was used to provide the uniform background. Locusts intermittently stop, walk, and hop as they advance in a common direction.

- https://youtu.be/kTuzFzH17Mo

2. Sample video with final processed data superimposed. Positions (circles) were obtained by automatic tracking. Heading directions (lines) were inferred from motion. Colors indicate motion state: stationary (red), walking (yellow), and hopping (green). Motion states were classified by a support vector machine operating on motion data from a local time window of each locusts trajectory.

- https://youtu.be/ASOetHW2eVk

\section{B Tables}

\begin{tabular}{crccc}
\hline & $\begin{array}{c}N \\
\text { (locusts) }\end{array}$ & $\begin{array}{c}\text { Density } \\
\left(\text { locusts } / \mathrm{m}^{2}\right)\end{array}$ & Polarization & Entropy Index \\
\hline all bands & 3369723 & $141.6 \pm 71.0$ & $0.82 \pm 0.062$ & $0.76 \pm 0.058$ \\
band 1 & 1135257 & $226.7 \pm 65.2$ & $0.84 \pm 0.040$ & $0.73 \pm 0.033$ \\
band 2 & 921094 & $145.9 \pm 19.1$ & $0.81 \pm 0.043$ & $0.77 \pm 0.023$ \\
band 3 & 979797 & $150.4 \pm 35.7$ & $0.80 \pm 0.059$ & $0.72 \pm 0.038$ \\
band 4 & 333575 & $55.5 \pm 21.4$ & $0.83 \pm 0.082$ & $0.82 \pm 0.058$ \\
\hline
\end{tabular}

Table 2: Indicators of collective marching for the full data set and divided by band. Typically, densities greater than 20 locusts $/ \mathrm{m}^{2}$ are associated with marching. Marching locusts have demonstrated polarization values in the range $0.6-0.9$ and entropy index values of $0.75-0.9$ [14]. Values are given as mean plus/minus one standard deviation. Definitions of density, polarization, and the entropy index are given in Measuring collective marching and a subsample of the full distributions is plotted in Figure 2. Note that values for each band are consistent with marching behavior. 


\begin{tabular}{cc|ccc|cccc}
\hline & \multirow{2}{*}{$\begin{array}{c}N \\
\text { (speeds) }\end{array}$} & \multicolumn{3}{|c|}{ Motion State (percent) } & \multicolumn{4}{c}{ Speed, mean $\pm \mathrm{SD}\left(\mathrm{cm} \mathrm{s}^{-1}\right)$} \\
& Stationary \% & Walking \% & Hopping \% & All & Stationary & Walking & Hopping \\
\hline all bands & 3332137 & 26.0 & 24.7 & 49.2 & $6.5 \pm 8.4$ & $0.2 \pm 0.7$ & $2.7 \pm 2.2$ & $11.8 \pm 9.3$ \\
band 1 & 1119569 & 12.2 & 26.2 & 61.6 & $8.4 \pm 8.8$ & $0.2 \pm 0.8$ & $3.1 \pm 2.3$ & $12.4 \pm 9.1$ \\
band 2 & 914361 & 39.9 & 28.1 & 32.0 & $4.1 \pm 6.5$ & $0.1 \pm 0.5$ & $2.6 \pm 2.0$ & $10.4 \pm 8.1$ \\
band 3 & 968173 & 28.8 & 18.5 & 52.7 & $6.3 \pm 8.9$ & $0.2 \pm 0.9$ & $2.1 \pm 2.1$ & $11.1 \pm 10.0$ \\
band 4 & 330034 & 26.6 & 28.7 & 44.7 & $7.4 \pm 8.8$ & $0.1 \pm 0.4$ & $3.2 \pm 2.3$ & $14.4 \pm 8.9$ \\
\hline
\end{tabular}

Table 3: Percent of data in each motion state and speeds divided by motion state for the complete data set and for each band. The percent of motion states varies significantly by band, probably due to environmental conditions such as temperature, but there is consistently a significant portion in each motion state. Speeds are given as mean plus/minus one standard deviation. Full distributions of speed are plotted as histograms in Figure 1 (left) and Figure 3.

\begin{tabular}{cc|cccc|ccccc}
\hline \multirow{2}{*}{ All bands } & \multirow{2}{*}{$\begin{array}{c}\text { N } \\
\text { (angles) }\end{array}$} & \multicolumn{3}{|c|}{ Two-fold anisotropy, $p=1$} & \multicolumn{4}{c}{ Four-fold anisotropy, $p=2$} \\
& $\left|\mathbf{M}_{1}\right|$ & $\phi_{1}$ & $-M_{1}^{s}$ & $M_{1}^{c}$ & $\left|\mathbf{M}_{2}\right|$ & $\phi_{2}$ & $-M_{2}^{s}$ & $M_{2}^{c}$ \\
\hline All Motion States & 6572930 & 0.0119 & -0.5341 & 0.0061 & 0.0102 & 0.0282 & -0.0060 & 0.0002 & 0.0282 \\
Stationary & 1505672 & 0.0040 & -2.5370 & $\mathbf{0 . 0 0 2 3}$ & -0.0033 & 0.0146 & -0.2191 & 0.0032 & $\mathbf{0 . 0 1 4 2}$ \\
Walking & 1527233 & 0.0173 & -1.5242 & $\mathbf{0 . 0 1 7 3}$ & 0.0008 & 0.0248 & -0.0670 & 0.0017 & $\mathbf{0 . 0 2 4 7}$ \\
Hopping & 3536882 & 0.0203 & -0.1393 & $\mathbf{0 . 0 0 2 8}$ & 0.0201 & 0.0358 & 0.0492 & -0.0018 & $\mathbf{0 . 0 3 5 7}$ \\
\hline
\end{tabular}

Table 4: Trigonometric moments computed from distributions of angles of relative neighbor positions within $7 \mathrm{~cm}$ of focal locusts in each motion state, see Quantifying anisotropy in neighbor density for full definitions. The number of separated concentrations is given by $p$. Overall anisotropy is characterized by $\left|\mathbf{M}_{p}\right|$ with direction given by $\phi_{p}$ (and $\phi_{p}+2 \pi / p$ ). Anisotropy aligned with the focal locust's heading direction is given $-M_{p}^{s}$ and perpendicular to the focal's heading is given by $-M_{p}^{c}$. Thus front-back asymmetry is measured by $-M_{1}^{s}$ and four-fold anisotropy perpendicular to the direction of motion is measured by $M_{2}^{c}$. Bolded values correspond to features we plot against the distance from the focal locust in Figure 5. Overall anisotropy $\left|\mathbf{M}_{p}\right|$ is significantly lower around stationary locusts than either moving state (for both $p$ values). The angle $\phi_{1}$ is approximately $-\pi / 2$ around walking locusts, indicating that the primary two-fold anisotropy is front-back. The angle $\phi_{2}$ is approximately 0 around both walking and hopping locusts, indicating that the primary four-fold anisotropy is perpendicular to the heading direction of the focal individual.

\section{Code}

The code used to extract, clean, and process the trajectory data, to conduct the analysis, and to generate the figures and tables is available on the public GitHub repository:

- https://github.com/weinburd/locust_trajectory_data

This repository has three levels of functionality.

1. By downloading only this repository, one can immediately run scripts to generate the figures and tables appearing in this paper from processed data stored in the repository itself.

2. By downloading this repository and the full data set in Data, one can recreate the figures and tables directly from the final data set used in our analysis. Additionally, this option enables the user to implement our data cleaning and processing pipeline on a pair of example data sets and evaluate the tracking accuracy agains our manually tracked ground-truth data. 
3. By downloading this repository, the full data set, and installing Fiji on the users own computer, one can execute the examples described in (2) including the tracking process to extract numerical data from example video files. For this option, the user will need to ensure that their Fiji installation includes the TrackMate v6.0.3 plugin and enable the Matlab-ImageJ update site.

\section{Data}

Upon publication, the final data set will be available at the Dryad repository:

- https://doi.org/10.5061/dryad.n02v6wwzz

The numerical trajectory data was extracted from video, cleaned, processed, and saved in Matlab's .mat format. The repository also contains two subdirectories. The first has example material where one can execute our full data extraction, cleaning, and processing pipeline. This material includes the starting preprocessed video, intermediate processed video and .xml data files, and final example data. The second subdirectory contains the two ground-truth data sets obtained by manual tracking in TrackMate. The original video files are also included so that the manually-tracked .xml files can be opened in Fiji $>$ Plugins $>$ Tracking $>$ Load a TrackMate File. 